\title{
Integrated mRNAseq and microRNAseq data analysis for grade III gliomas
}

\author{
JUNQIANG DAI $^{*}$, ZHITONG BING ${ }^{2 *}$, YINIAN ZHANG ${ }^{1}$, QIAO LI ${ }^{1}$, LIANG NIU ${ }^{1}$, \\ WENTAO LIANG ${ }^{1}$, GUOQIANG YUAN ${ }^{3}$, LEI DUAN ${ }^{1}$, HANG YIN $^{1}$ and YAWEN PAN ${ }^{1,3}$ \\ ${ }^{1}$ Department of Neurosurgery, Lanzhou University Second Hospital, Lanzhou, Gansu 730030; \\ ${ }^{2}$ Institute of Modern Physics, Chinese Academy of Sciences, Lanzhou, Gansu 730000; ${ }^{3}$ Institute of \\ Neurology, Lanzhou University Second Hospital, Lanzhou, Gansu 730030, P.R. China
}

Received August 10, 2016; Accepted July 6, 2017

DOI: $10.3892 / \mathrm{mmr} .2017 .7545$

\begin{abstract}
The World Health Organization classification distinguishes four grades for gliomas. Grade III gliomas, which are brain malignant brain tumors with variable biological behavior and propensity, have been not widely investigated. The objective of the present study was to identify specific gene modules and valuable hubs associated with gliomagenesis and molecular signatures to assist in determining grade III glioma prognosis. mRNAseq and micro (mi)RNAseq data were used to construct a co-expression network of gliomas using weight gene co-expression network analysis, and revealed the prognostic molecular signature of grade III gliomas. The differently expressed miRNAs and mRNAs were identified. A total of 37 mRNAs and 10 miRNAs were identified, which were closely associated with the survival rates of patients with grade III glioma. To further understand the tumorigenesis, Cytoscape software was used to construct a network containing these differently expressed molecules. The result suggested that both the downregulated genes and upregulated genes are vital in the process of glioma deterioration, and certain genes are closely associated with clinical prognosis.
\end{abstract}

\section{Introduction}

Gliomas are common primary tumors of the central nervous system. According to the World Health Organization classification, grade I and II gliomas are benign tumors, whereas grade III gliomas are a class of malignant brain solid tumor with a median patient survival rate of 2-5 years (1). These

Correspondence to: Professor Yawen Pan, Department of Neurosurgery, Lanzhou University Second Hospital, 82 Cuiyingmen Road, Chengguan, Lanzhou, Gansu 730030, P.R. China

E-mail: panyawen666@sohu.com

*Contributed equally

Key words: lower grade glioma, microRNAseq, mRNAseq, weight gene co-expression network analysis, the cancer genome atlas represent $10 \%$ of primary brain tumors (2), which can infiltrate the surrounding brain parenchyma. Using standard therapeutic protocols, patients with malignant glioma have different pathological appearances and clinical outcomes. Treatments include surgery, radiation therapy, and chemotherapy, however, there is no specialized treatment available. There are insufficient molecular targets relevant in the choice of therapy, and their role in clinical trials requires validation. Novel therapeutic methods based on the specific mechanism of high grade glioma carcinogenesis are required to improve treatment efficiency and avoid the side effects of traditional treatment.

In order to better understand the mechanisms underlying complicated diseases, building and analyzing biological networks associated with the intractable diseases are becoming an efficient approach. Instead of individual genetic determinants, network approaches provide an insight into the pathogenesis of complex diseases by examining interacting gene sets and pathways $(3,4)$. The network analysis of expression profile data has been able to identify genes modules associated with tumorigenesis. In addition, this method can be used to understand the mechanisms underlying gliomagenesis at the system and gene level.

Weighted gene co-expression network analysis (WGCNA) was been widely used to examine the changes of transcriptome expression patterns in various diseases, which identifies clusters (modules) of highly correlated genes, and summarizes clusters using the module eigengene or an intramodular hub gene (5-8). Correlation networks facilitate network-based gene screening methods, which can be used to identify candidate biomarkers or therapeutic targets. These methods have been successfully applied in complex biological contexts, including cancer, mouse genetics, yeast and genetics, and the analysis of brain imaging data (7). In addition, the algorithm of WGCNA can simplify the problems of multiple testing, which are unavoidable in standard gene-centric methods of microarray expression profiling data analysis; consequently it is a useful systematic analysis method, which focuses on the coherence function of network modules (9).

The Cancer Genome Atlas (TCGA) project has provided a comprehensive means to improve the ability to diagnose, treat and prevent cancer through an improved understanding 
of the genetic basis of the disease. By the end of 2015, TCGA had analyzed the genomic, epigenomic and gene expression profiles of $>10,000$ specimens from $>25$ types of tumor (10). This substantial data provides opportunities to identify the mechanism and prognostic molecular signatures of cancer in a comprehensive manner. The lower grade glioma (LGG) group data in TCGA includes the grade II and grade III glioma gene expression profiles and relevant clinical data of those samples. To better understand the mechanism underlying clinical heterogeneity, the present study combined the LGG micro (mi)RNAseq and mRNAseq data of TCGA to identify the relevant network of tumorigenesis and prognostic genes in clinical traits.

\section{Materials and methods}

Patient characteristics and integrated mRNA and miRNA profiles. The clinical, and mRNAseq and miRNAseq data for 83 patients with grade III glioma were downloaded from the LGG cohort. The correspondent normal cohort data were obtained from five TCGA glioblastoma (GBM) normal control samples. The TCGA-Assembler download level-3 RNASeqV2 gene expression data, miRNA-seq data of samples and the clinical information of the patients were used (DirectoryTraverseResult_Sep-18-2015.rda). The raw count mRNAseq data of 83 glioma grade III patients and raw read miRNA data of five GBM normal patients (TCGA-06-AABW11A-31R-A36H-07, TCGA-06-0678-11A-32R-A36H-07, TCGA-06-0675-11A-32R-A36H-07, TCGA-06-0681-11A41R-A36H-07, TCGA-06-0680-11A-32R-A36H-07 and TCGA-HW-7493-01A-11R-2027-07), were selected.

Statistical analyses. Expressed data close to zero were eliminated, and round numbers of all arrays were selected. The normal group were compared with the grade III glioma group, and the 'DESeq' package in R software (3.3.0; www.r-project. org) was used to identify the differentially expressed genes (DEGs), miRNAs with a fold change $>2.0$, and adjusted $\mathrm{P}$-value of $\mathrm{P}<0.05$.

The WGCNA was used to identify the co-expression modules $(5,7,11)$. WGCNA was implemented in the Bioconductor package (bioconductor.org/biocLite.R).

The DEGs were applied to identify the gene modules of highly correlated genes using WGCNA. A total of 2,036 of the DEGs were selected and Pearson's correlation was calculated for all pairs of selected genes. The correlation matrix was converted into an adjacency matrix with a power function, so that the connection strength between two genes, $x_{i}$ and $x_{j}$, was defined as: $a_{i j}=\left|0.5 x\left(1+\operatorname{cor}\left(x_{i}, x_{j}\right)\right)\right|^{\beta}$. Where $x_{i}$ and $x_{j}$ represent the expression values of probes, and parameter $\beta$ was determined by the criterion that the resulting adjacency matrix approximately fit a scale-free topological feature according to a previously proposed model-fitting index (11). The row index $\mathrm{u}(\mathrm{u}=1, \ldots, \mathrm{m})$ represents sample measurements. The adjacency matrix was further transformed into a topological overlap matrix, which captures not only the direct interaction between two genes, but also their indirect interactions through all the other genes in the network. In the present study, two functions of adjacency matrices were defined. First, the Topological Overlap Matrix (TOM) is defined as follows:

$$
\begin{gathered}
\operatorname{TOM}_{i j}=\frac{\sum_{u} a_{i u} a_{u j}+a_{i j}}{\min \left(k_{i}, k_{j}\right)+1-a_{i j}} \\
k_{i}=\sum_{u} a_{i u}
\end{gathered}
$$

where is the node connectivity. A second function was used as a distance matrix in the hierarchical clustering of the transcript units for module detection, and was defined as follows:

$$
\operatorname{Dissim}_{i j}=1-\text { TOM }_{i j}
$$

Using the clusterProfiler package of Bioconductor, the functions of different module genes were annotated by gene ontology (GO; www.geneontology.org) and Kyoto Encyclopedia of Genes and Genomes (KEGG; www.genome.jp/kegg) analysis. Finally, the co-expression network of DEGs was established and visualized using Cytoscape software. For clarifying the role of miRNAs in grade III glioma, the differentially expressed miRNA data and mRNA data were merged to construct the co-expression network.

In order to identify prognostic mRNA and miRNA signatures, by combining the clinical data of the patient hub genes in TCGA, life curves were constructed for those samples with DEGs by 'survival' in R package. All analyses were performed using R software (version 3.3.0) and Bioconductor (version 3.2).

\section{Results}

DEGs. A total of 2,036 differently expressed mRNAs and 50 miRNAs were confirmed using the 'DESeq' package in R. The heatmap constructed using the differently expressed mRNAs is shown in Fig. 1. The mRNAs with $\mid \log 2$ foldchangel $>2$ and miRNAs with $\mid \log 2$ foldchangel $>2$ are shown in Tables I and II.

Hub genes. The 2,036 genes were clustered into five modules (Fig. 2) using WGCNA. In addition, the co-expression network of DEGs was established using WGCNA and visualized using Cytoscape software. In the network, BUB1B, KIFC1, TOP2A, BUB1, SLC12A5, ESCO2, ESPL1, EPR1, KIF15, CASC5, SGOL1, NUSAP1, CCNB2, NUF2, TTK and KIF2C were central in the network (Fig. 3). It was found that the network included two centers, with downregulated genes and upregulated genes constituting the regulatory network, respectively. BUB1B, KIFC1, TOP2A, BUB1, ESPL1 and EPR1 were at the center of the upregulated gene network; SLC12A5, VSNL1, SULT4A1, TMEM130, SNAP25 were central of the downregulated expression gene network. However, when the data of the differently regulated mRNAseq and miRNAseq were merged to construct the co-expression network, SLC12A5, MAL2, VSNL1, A2BP1, EPB49, SULT4A1, TMEM130, ADAM11, SNAP25, Clorf115, DNM1 and SYT1 were central in the network, and miR-128 and miR-129 were involved. (Fig. 4). It was hypothesized that the genes in the center of the network may be hub genes in the pathological process of high grade LGG.

Functional analysis. The present study identified the top eight GO biological processes of the five gene modules (Table III), and performed KEGG analysis (Table IV). The pathway 
Table I. Differential expression of miRNAs between the glioma and normal groups.

\begin{tabular}{|c|c|c|c|}
\hline ID & log2foldchange & pval & padj \\
\hline hsa-miR-137 & -3.52 & $<0.01$ & $<0.01$ \\
\hline hsa-miR-876 & -3.18 & $<0.01$ & $<0.01$ \\
\hline hsa-miR-433 & -2.92 & $<0.01$ & $<0.01$ \\
\hline hsa-miR-218-2 & -2.85 & $<0.01$ & $<0.01$ \\
\hline hsa-miR-485 & -2.83 & $<0.01$ & $<0.01$ \\
\hline hsa-miR-873 & -2.81 & $<0.01$ & 0.02 \\
\hline hsa-miR-448 & -2.67 & $<0.01$ & 0.01 \\
\hline hsa-miR-770 & -2.67 & $<0.01$ & 0.01 \\
\hline hsa-miR-329-2 & -2.66 & $<0.01$ & 0.03 \\
\hline hsa-miR-329-1 & -2.63 & $<0.01$ & 0.03 \\
\hline hsa-miR-495 & -2.60 & $<0.01$ & $<0.01$ \\
\hline hsa-miR-656 & -2.60 & $<0.01$ & 0.02 \\
\hline hsa-miR-412 & -2.58 & $<0.01$ & $<0.01$ \\
\hline hsa-miR-668 & -2.48 & $<0.01$ & 0.03 \\
\hline hsa-miR-138-2 & -2.46 & $<0.01$ & $<0.01$ \\
\hline hsa-miR-7-3 & -2.45 & $<0.01$ & 0.02 \\
\hline hsa-miR-139 & -2.43 & $<0.01$ & $<0.01$ \\
\hline hsa-miR-129-2 & -2.43 & $<0.01$ & $<0.01$ \\
\hline hsa-miR-487b & -2.39 & $<0.01$ & 0.01 \\
\hline hsa-miR-129-1 & -2.39 & $<0.01$ & $<0.01$ \\
\hline hsa-miR-1298 & -2.38 & $<0.01$ & 0.03 \\
\hline hsa-miR-1224 & -2.37 & $<0.01$ & 0.02 \\
\hline hsa-miR-380 & -2.36 & $<0.01$ & 0.04 \\
\hline hsa-miR-889 & -2.33 & $<0.01$ & 0.01 \\
\hline hsa-miR-432 & -2.27 & $<0.01$ & 0.01 \\
\hline hsa-miR-490 & -2.26 & $<0.01$ & 0.03 \\
\hline hsa-miR-1258 & -2.25 & $<0.01$ & 0.05 \\
\hline hsa-miR-543 & -2.22 & $<0.01$ & 0.05 \\
\hline hsa-miR-323 & -2.18 & $<0.01$ & 0.01 \\
\hline hsa-miR-431 & -2.16 & $<0.01$ & $<0.01$ \\
\hline hsa-miR-138-1 & -2.03 & $<0.01$ & 0.03 \\
\hline hsa-miR-410 & -2.00 & $<0.01$ & 0.05 \\
\hline hsa-miR-10b & 8.59 & $<0.01$ & $<0.01$ \\
\hline hsa-miR-891b & 5.35 & $<0.01$ & 0.05 \\
\hline hsa-miR-181a-2 & 2.49 & $<0.01$ & $<0.01$ \\
\hline hsa-miR-92b & 2.13 & $<0.01$ & 0.03 \\
\hline hsa-miR-27a & 2.05 & $<0.01$ & 0.05 \\
\hline hsa-miR-23a & 2.015 & $<0.01$ & 0.05 \\
\hline hsa-miR-374a & 1.91 & $<0.01$ & 0.05 \\
\hline hsa-miR-25 & 1.68 & $<0.01$ & 0.05 \\
\hline
\end{tabular}

miR, microRNA.

enrichment analysis combined several physiological and pathological processes of the nervous system. The genes of the turquoise module were downregulated in glioma; in addition, GO and KEGG analysis predicted that these genes were involved in several important physiological processes in the central nervous system. However, the brown module included genes, which were upregulated in glioma, and GO and KEGG
Table II. Differential expression of mRNAs between the glioma and normal groups.

\begin{tabular}{|c|c|c|c|}
\hline ID & $\log 2 \mathrm{FC}$ & pval & padj \\
\hline INS & -7.90 & $<0.01$ & 0.05 \\
\hline LOC100129935 & -5.91 & $<0.01$ & 0.01 \\
\hline TRIM43 & -5.21 & $<0.01$ & 0.04 \\
\hline FAM153B & -5.13 & $<0.01$ & $<0.01$ \\
\hline LOC440896 & -5.09 & $<0.01$ & $<0.01$ \\
\hline MSLNL & -5.04 & $<0.01$ & $<0.01$ \\
\hline FAM153C & -4.99 & $<0.01$ & $<0.01$ \\
\hline FAM153A & -4.94 & $<0.01$ & $<0.01$ \\
\hline C6orf127 & -4.87 & $<0.01$ & $<0.01$ \\
\hline KRT77 & -4.79 & $<0.01$ & 0.02 \\
\hline LOC728276 & -4.69 & $<0.01$ & $<0.01$ \\
\hline EVPLL & -4.67 & $<0.01$ & 0.05 \\
\hline LOC100132354 & -4.61 & $<0.01$ & $<0.01$ \\
\hline KRTAP17-1 & -4.58 & $<0.01$ & 0.03 \\
\hline ANXA8 & -4.38 & $<0.01$ & 0.05 \\
\hline MYH13 & -4.31 & $<0.01$ & $<0.01$ \\
\hline CRYGN & -4.29 & $<0.01$ & 0.03 \\
\hline CRHR2 & -4.22 & $<0.01$ & $<0.01$ \\
\hline KIF12 & -4.21 & $<0.01$ & $<0.01$ \\
\hline GPR150 & -4.19 & $<0.01$ & $<0.01$ \\
\hline KRT33B & -4.17 & $<0.01$ & $<0.01$ \\
\hline ADRB3 & -4.14 & $<0.01$ & $<0.01$ \\
\hline SLC22A10 & -4.13 & $<0.01$ & $<0.01$ \\
\hline KRT3 & -4.10 & $<0.01$ & 0.02 \\
\hline FSHB & -4.09 & $<0.01$ & 0.02 \\
\hline MYL2 & -4.07 & $<0.01$ & 0.01 \\
\hline HOXD9 & 9.01 & $<0.01$ & 0.04 \\
\hline TLX1 & 8.91 & $<0.01$ & 0.04 \\
\hline TBX5 & 8.14 & $<0.01$ & $<0.01$ \\
\hline HOXD8 & 7.67 & $<0.01$ & $<0.01$ \\
\hline PAX1 & 6.94 & $<0.01$ & $<0.01$ \\
\hline TOP2A & 6.81 & $<0.01$ & $<0.01$ \\
\hline VEPH1 & 6.71 & $<0.01$ & $<0.01$ \\
\hline C5orf38 & 6.71 & $<0.01$ & $<0.01$ \\
\hline DLGAP5 & 6.57 & $<0.01$ & $<0.01$ \\
\hline MYBL2 & 6.46 & $<0.01$ & $<0.01$ \\
\hline GSC & 6.38 & $<0.01$ & 0.03 \\
\hline PBK & 6.28 & $<0.01$ & $<0.01$ \\
\hline UBE2C & 6.19 & $<0.01$ & $<0.01$ \\
\hline CDC45 & 6.13 & $<0.01$ & $<0.01$ \\
\hline NDC80 & 6.04 & $<0.01$ & $<0.01$ \\
\hline MELK & 5.98 & $<0.01$ & $<0.01$ \\
\hline AURKB & 5.94 & $<0.01$ & $<0.01$ \\
\hline ZNF560 & 5.92 & $<0.01$ & 0.04 \\
\hline RRM2 & 5.84 & $<0.01$ & $<0.01$ \\
\hline FAM64A & 5.82 & $<0.01$ & $<0.01$ \\
\hline IRX1 & 5.79 & $<0.01$ & $<0.01$ \\
\hline CCNB2 & 5.76 & $<0.01$ & $<0.01$ \\
\hline MKI67 & 5.69 & $<0.01$ & $<0.01$ \\
\hline TSHR & 5.68 & $<0.01$ & 0.04 \\
\hline KIF20A & 5.66 & $<0.01$ & $<0.01$ \\
\hline NCAPG & 5.51 & $<0.01$ & $<0.01$ \\
\hline
\end{tabular}




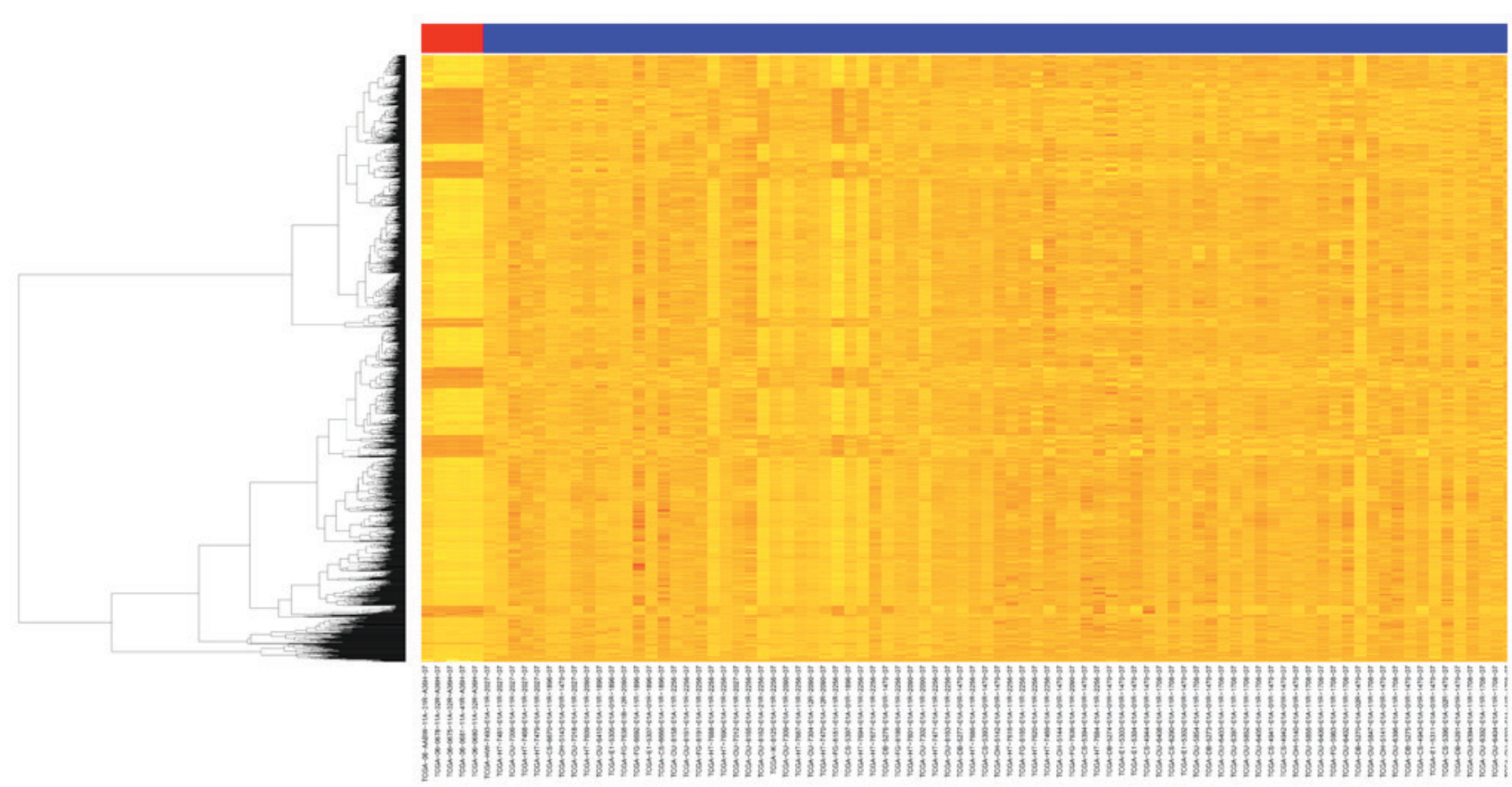

Figure 1. Hierarchical clustering of mRNAs. Differences in mRNA expression between the glioma group (blue color) and normal group (red color) were visualized by clustering of sample data using the gplots package in R language. Light yellow represents an expression level above the mean; dark orange colors represent expression below the mean.

analysis showed these were involved in important pathways, including cell cycle and tumorigenesis.

Clinical biomarkers. Finally, the present study identified the gene symbols associated with clinical outcome (Table V). A total of eight prognostic RNA signatures were found (Fig. 5). C1orf115, CACS5, CDC45, DLL3, EPR1, HOXD9, KIF20, KIF4A, KIF14, KLK7, MELK, NCAPG, PBK, RASL1, SGOL1, SNAP25, SULT4A1, TMEM130, TSHR and VEPH1 were significantly associated with clinical survival rates $(\mathrm{P}<0.05)$. In addition, certain genes were associated with LGG patient prognosis $(0.05<\mathrm{P}<0.1)$, including A2BP, AURKB, CRHR2, HIPK4, HJURP, MIK67, MYBL2, RRM2, SPARC, TOP2A and VSNL1. When the miRNAseq data and clinical information of samples were combined, it was found that has-miR-10b, has-miR-27a, has-miR-138-2, has-miR-138-1, has-miR-139, has-miR-329-1, has-miR-412, has-miR-431, has-miR-495 and has-miR-656 were also closely associated with LGG patient outcome and may be prognostic miRNA signatures (Table VI). The survival curves of has-miR-10b, has-miR-27a, has-miR-138-2 and has-miR-329-1 are shown in Fig. 6.

In the present study, miR-10b and miR-27a were expressed at high levels in glioma tissue, and the expression levels were associated with poor overall survival rates in patients with high grade gliomas. A number of downregulated miRNAs, including has-miR-138-2, has-miR-138-1, has-miR-139 and has-miR-329-1, were also associated with outcome in patients with glioma.

\section{Discussion}

Malignant gliomas are the most common and life-threatening type of primary intracranial tumor, which include anaplastic

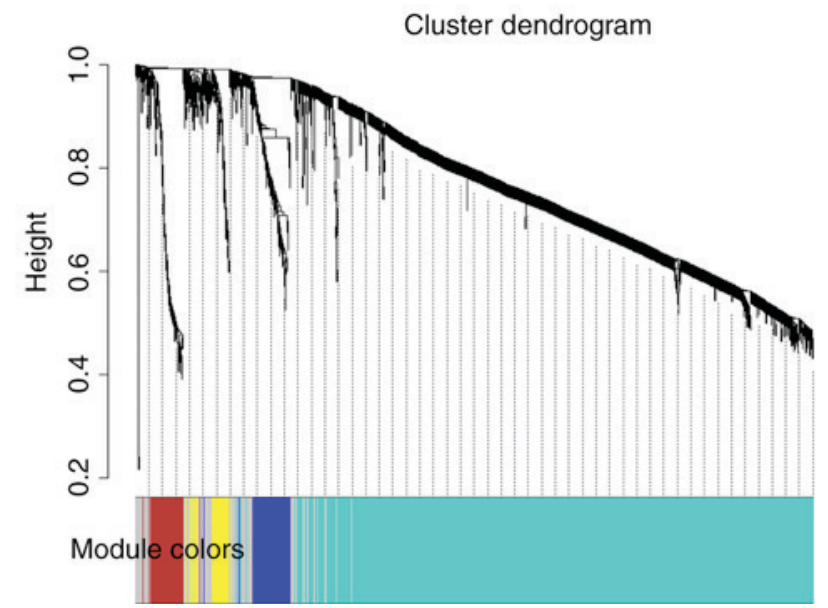

Figure 2. Network analysis of gene expression. Network analysis identified five distinct modules in lower grade glioma. The dendrogram was produced by average linkage hierarchical clustering of 2,036 genes based on weighted gene co-expression network analysis. The different colors represent different clustering modules.

astrocytoma, anaplastic oligodendroglioma and GBM. Several efforts have been made to identify the key regulatory genes or molecules in these types of malignant tumor. However, to the best of our knowledge, few studies have been performed to predict the prognosis of grade III gliomas, and no reliable biomarkers for the detection and risk stratification of gliomas have been identified.

BUB1B/BubR1, a protein that monitors proper spindle microtubule attachment to the kinetochore, has been found to be a promising candidate for targeted therapies in GBM $(12,13)$. In the present study, BUB1B was overexpressed in glioma tissues, and was associated with the clinical outcome 

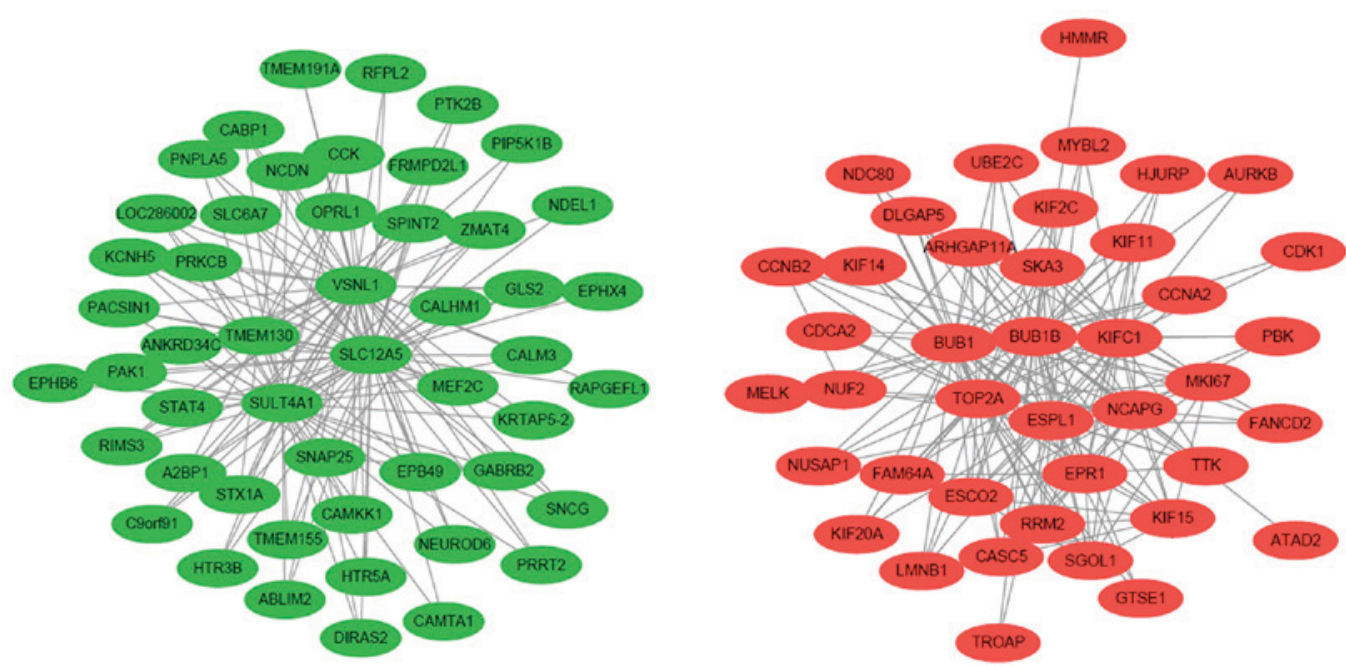

Figure 3. Network of differently expressed mRNAs constructed using weighted gene co-expression network analysis in low grade glioma, and visualized using Cytoscape software. BUB1B, KIFC1, TOP2A, BUB1, ESPL1 and EPR1 were central in the upregulated gene network; SLC12A5, VSNL1, SULT4A1, TMEM130 and SNAP25 were central in downregulated gene network. Red represents an expression level above the mean; green represents expression below the mean. Lines illustrate the correlations between genes.

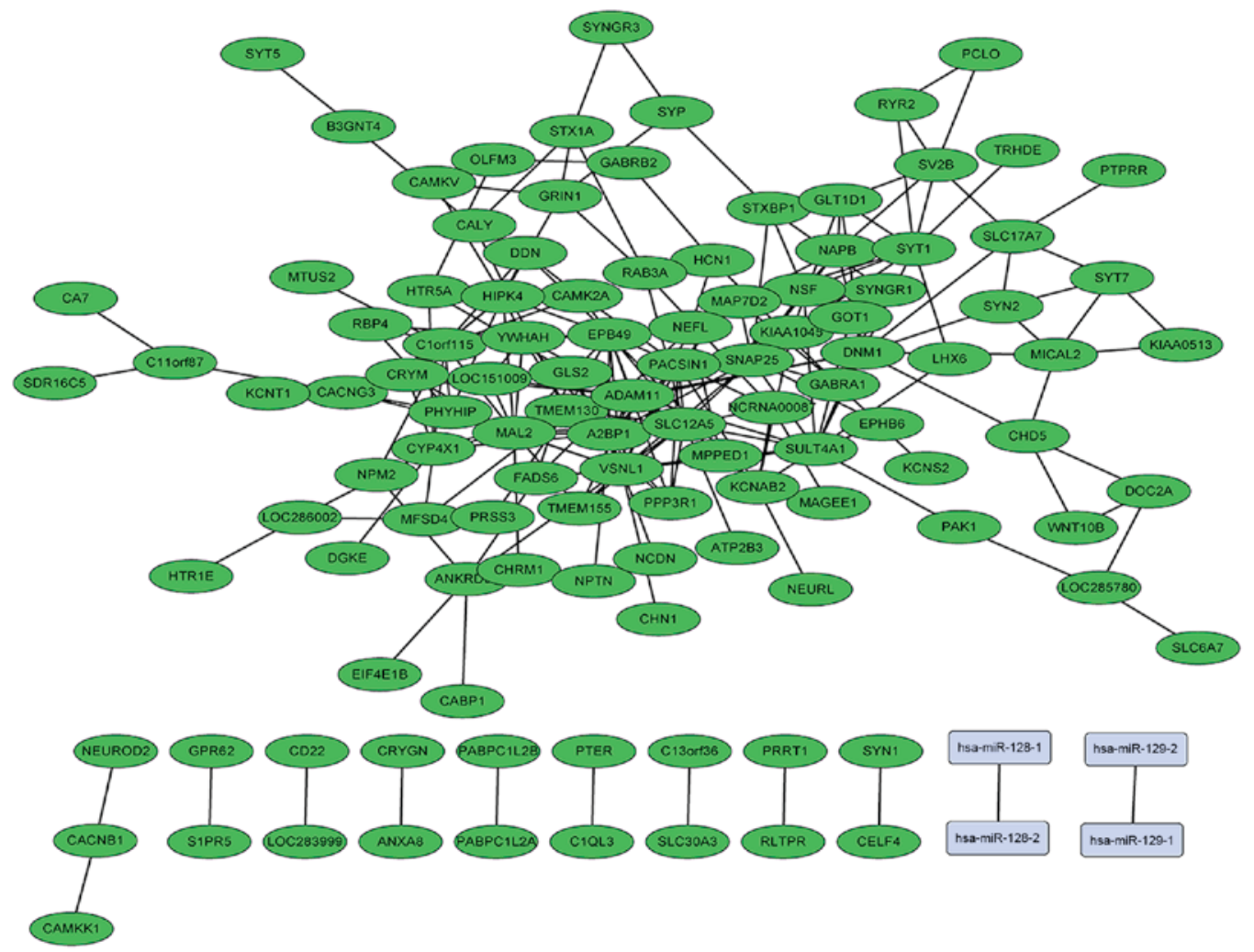

Figure 4. Network of different expressed mRNAs and microRNAs constructed using weighted gene co-expression network analysis in low grade glioma visualized using Cytoscape software. SLC12A5, MAL2, VSNL1, A2BP1, EPB49, SULT4A1, TMEM130, ADAM11, SNAP25, C1orf115, DNM1 and SYT1 were central in the network. Green represents downregulated genes. Lines illustrate the correlations between genes. miR-128 and miR-129 were involved. miR, microRNA.

of patients with glioma $(\mathrm{P}=0.06)$. Patients with a high expression of BUB1B had shorter survival rates. In the co-expression network, BUB1B was located centrally in the network, and the results suggested that BUB1B may be a potential target for high grade glioma.

The kinesin motor KIFC1 has been suggested as a potential chemotherapeutic target due to its importance in the clustering of multiple centrosomes found in cancer cells (14). However, the function of KIFC1 in high grade gliomas remain to be elucidated. The present study found that KIFC1 was upregulated in grade III glioma tissues and located centrally in the network. Further investigations are required to annotate its effect on brain tumors.

Topoisomerase 2A (TOP2A) is overexpressed in proliferating cells $(15,16)$. The expression of TOP2A has been correlated with aggressive and highly proliferative types 
Table III. GO enrichment analysis in gene modules of the top eight significantly enriched biology terms.

\begin{tabular}{|c|c|c|c|c|}
\hline Module & P-value & ID & Ontology & Term name \\
\hline Blue & 0.001965 & GO:0015631 & MF & Tubulin binding \\
\hline Blue & 0.003507 & GO:0017034 & MF & Rap guanyl-nucleotide exchange factor activity \\
\hline Blue & 0.008008 & GO:0010008 & $\mathrm{CC}$ & Endosome membrane \\
\hline Blue & 0.009040 & GO:0005768 & $\mathrm{CC}$ & Endosome \\
\hline Blue & 0.010111 & GO:0003376 & $\mathrm{BP}$ & Sphingosine-1-phosphate signaling pathway \\
\hline Blue & 0.010111 & GO:0015693 & $\mathrm{BP}$ & Magnesium ion transport \\
\hline Blue & 0.010111 & GO:0018345 & $\mathrm{BP}$ & Protein palmitoylation \\
\hline Blue & 0.010111 & GO:0031365 & $\mathrm{BP}$ & N-terminal protein amino acid modification \\
\hline Brown & $4.57 \mathrm{E}-38$ & GO:1903047 & $\mathrm{BP}$ & Mitotic cell cycle process \\
\hline Brown & $3.76 \mathrm{E}-37$ & GO:0000278 & $\mathrm{BP}$ & Mitotic cell cycle \\
\hline Brown & $6.10 \mathrm{E}-37$ & GO:0022402 & $\mathrm{BP}$ & Cell cycle process \\
\hline Brown & $1.17 \mathrm{E}-36$ & GO:0007049 & $\mathrm{BP}$ & Cell cycle \\
\hline Brown & $6.96 \mathrm{E}-34$ & GO:0007067 & $\mathrm{BP}$ & Mitotic nuclear division \\
\hline Brown & $2.90 \mathrm{E}-33$ & GO:0007059 & $\mathrm{BP}$ & Chromosome segregation \\
\hline Brown & $1.24 \mathrm{E}-29$ & GO:0051301 & $\mathrm{BP}$ & Cell division \\
\hline Brown & $4.40 \mathrm{E}-25$ & GO:0005694 & $\mathrm{CC}$ & Chromosome \\
\hline Grey & $9.10 \mathrm{E}-10$ & GO:0009888 & $\mathrm{BP}$ & Tissue development \\
\hline Grey & 8.98E-09 & GO:0009887 & $\mathrm{BP}$ & Organ morphogenesis \\
\hline Grey & $1.98 \mathrm{E}-08$ & GO:0005578 & $\mathrm{CC}$ & Proteinaceous extracellular matrix \\
\hline Grey & $2.65 \mathrm{E}-08$ & GO:0048729 & $\mathrm{BP}$ & Tissue morphogenesis \\
\hline Grey & 4.74E-08 & GO:0031012 & $\mathrm{CC}$ & Extracellular matrix \\
\hline Grey & $6.47 \mathrm{E}-08$ & GO:0007389 & $\mathrm{BP}$ & Pattern specification process \\
\hline Grey & 8.30E-08 & GO:0001655 & $\mathrm{BP}$ & Urogenital system development \\
\hline Grey & $1.05 \mathrm{E}-07$ & GO:0060429 & $\mathrm{BP}$ & Epithelium development \\
\hline Turquoise & $5.13 \mathrm{E}-16$ & GO:0007268 & $\mathrm{BP}$ & Synaptic transmission \\
\hline Turquoise & $4.20 \mathrm{E}-14$ & GO:0045202 & $\mathrm{CC}$ & Synapse \\
\hline Turquoise & $3.08 \mathrm{E}-12$ & GO:0034220 & $\mathrm{BP}$ & Ion transmembrane transport \\
\hline Turquoise & $3.35 \mathrm{E}-12$ & GO:0006811 & $\mathrm{BP}$ & Ion transport \\
\hline Turquoise & $8.37 \mathrm{E}-11$ & GO:0007267 & $\mathrm{BP}$ & Cell-cell signaling \\
\hline Turquoise & $3.69 \mathrm{E}-10$ & GO:0098655 & $\mathrm{BP}$ & Cation transmembrane transport \\
\hline Turquoise & $6.46 \mathrm{E}-10$ & GO:0055085 & $\mathrm{BP}$ & Transmembrane transport \\
\hline Turquoise & $1.53 \mathrm{E}-09$ & GO:0042995 & $\mathrm{CC}$ & Cell projection \\
\hline Yellow & $7.08 \mathrm{E}-16$ & GO:0000184 & $\mathrm{BP}$ & $\begin{array}{l}\text { Nuclear-transcribed mRNA catabolic process, } \\
\text { nonsense-mediated decay }\end{array}$ \\
\hline Yellow & $7.60 \mathrm{E}-15$ & GO:0000956 & $\mathrm{BP}$ & Nuclear-transcribed mRNA catabolic process \\
\hline Yellow & $4.44 \mathrm{E}-14$ & GO:0006402 & $\mathrm{BP}$ & mRNA catabolic process \\
\hline Yellow & $1.88 \mathrm{E}-13$ & GO:0006401 & $\mathrm{BP}$ & RNA catabolic process \\
\hline Yellow & $2.09 \mathrm{E}-13$ & GO:0006413 & $\mathrm{BP}$ & Translational initiation \\
\hline Yellow & $2.44 \mathrm{E}-13$ & GO:0006613 & $\mathrm{BP}$ & Cotranslational protein targeting to membrane \\
\hline Yellow & $2.44 \mathrm{E}-13$ & GO:0006614 & $\mathrm{BP}$ & $\begin{array}{l}\text { SRP-dependent cotranslational protein } \\
\text { targeting to membrane }\end{array}$ \\
\hline Yellow & $8.38 \mathrm{E}-13$ & GO:0006412 & $\mathrm{BP}$ & Translation \\
\hline
\end{tabular}

GO, Gene ontology; MF, molecular function; CC, cellular component; BP, biological process.

of cancer $(17,18)$. In glioma, the levels of TOP2A have been reported as a proliferation marker in association with the Ki-67 index (19). The protein levels of TOP2A were correlated with survival rates in two previous studies, which noted that patients with improved survival rates had lower mean levels of TOP2A $(20,21)$. The data revealed that temozolomide inhibited the expression of TOP2A. In the present study, TOP2A was associated with the clinical outcome of patients with glioma $(\mathrm{P}=0.08)$ and may be a hub gene in gliomagenesis.

As with BUB1B, BUB1 is a major mitotic spindle assembly checkpoint gene and significantly correlates with glioma grade and survival rates (22). In the present study, the 
Table IV. Kyoto Encyclopedia of Genes and Genomes enrichment analysis of gene modules with the top eight significantly enriched biology terms.

\begin{tabular}{|c|c|c|c|c|}
\hline Module & ID & Description & Gene ratio & P-value \\
\hline Brown & hsa04110 & Cell cycle & $11 / 39$ & $<0.01$ \\
\hline Brown & hsa04114 & Oocyte meiosis & $7 / 39$ & $<0.01$ \\
\hline Brown & hsa04914 & Progesterone-mediated oocyte maturation & $6 / 39$ & $<0.01$ \\
\hline Brown & hsa04115 & p53 signaling pathway & $5 / 39$ & $<0.01$ \\
\hline Turquoise & hsa04080 & Synaptic vesicle cycle & $59 / 558$ & $<0.01$ \\
\hline Turquoise & hsa04020 & Glutamatergic synapse & $51 / 558$ & $<0.01$ \\
\hline Turquoise & hsa04921 & Retrograde endocannabinoid signaling & $47 / 558$ & $<0.01$ \\
\hline Turquoise & hsa04024 & Morphine addiction & $47 / 558$ & $<0.01$ \\
\hline Turquoise & hsa04010 & GABAergic synapse & $45 / 558$ & $<0.01$ \\
\hline Turquoise & hsa04724 & Calcium signaling pathway & $43 / 558$ & $<0.01$ \\
\hline Turquoise & hsa04723 & Oxytocin signaling pathway & $40 / 558$ & $<0.01$ \\
\hline Turquoise & hsa04728 & Circadian entrainment & $38 / 558$ & $<0.01$ \\
\hline Turquoise & hsa04728 & Circadian entrainment & $38 / 558$ & $<0.01$ \\
\hline
\end{tabular}
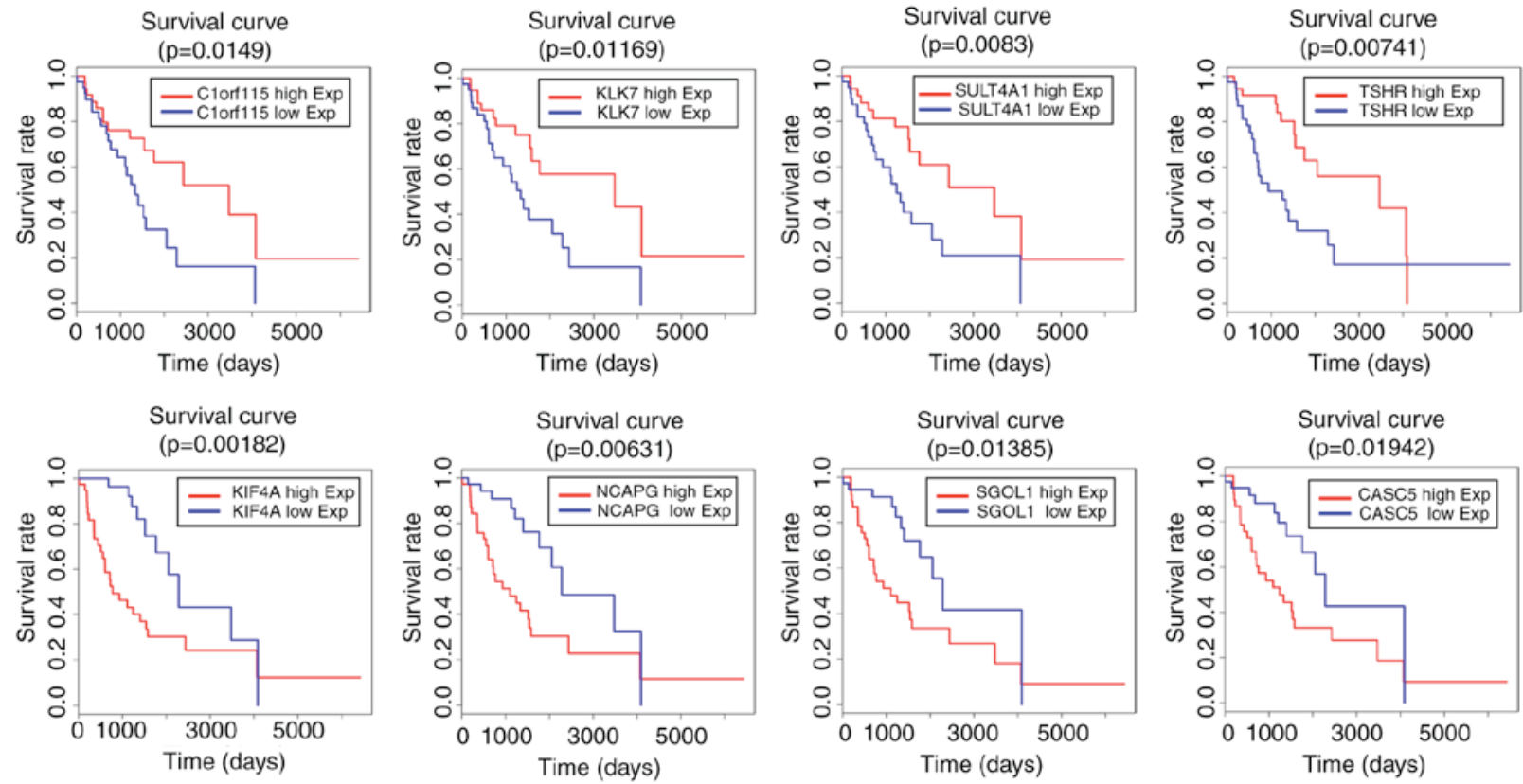

Figure 5. Genes predicting the prognosis of patients with glioma. Downregulated genes KIF4A, NCAPG, SGOL1 and CASC5 predicted increased survival rate; upregulated genes C1orf115, KLK7, SULT4A1 and TSHR predicted increased survival rate. Exp, expression.

patients with overexpression of the BUB1 gene had a shorter survival rate $(\mathrm{P}=0.1)$. In colorectal cancer, mutation of the BUB1 gene was found to be associated with lymph node metastasis and lower relapse-free survival rates following surgery (23). Further investigations may be required to identify whether BUB1 mutations are important in the glioma process. In addition, the expression of ESPL1 in human glioma and its possible correlations with histoclinical features remains to be fully elucidated, however, evidence suggests that ESPL1 is a candidate oncogene in breast cancer and lung cancer $(24,25)$.

SLC12A5 has been found have an important oncogenic role in colorectal carcinogenesis; its overexpression can be an independent prognostic factor for patients, and the mutation frequency of SLC12A5 may have potential oncogenic effects in colon cancer $(26,27)$. However, the functional characterization of SLC12A5 in brain tumors remains to be fully elucidated, and few investigations have been performed. The present study found that patients with a high expression of SLC12A5 showed improved prognosis $(\mathrm{P}=0.11)$. Further investigations are required to clarify the function of SLC12A5 in glioma and other brain tumors.

VSNL1 is a known tumor-suppressor gene regulating cell migration in several types of cancer. It is also downregulated in GBM (28). The data obtained in the present study suggested that the overexpression of VSNAL1 may be associated with 
Table V. Gene symbols associated with improved clinical outcome.

\begin{tabular}{|c|c|}
\hline Gene & P-value \\
\hline \multicolumn{2}{|c|}{ Downregulated } \\
\hline KIF4A & 0.01 \\
\hline NCAPG & 0.01 \\
\hline SGOL1 & 0.01 \\
\hline CASC5 & 0.02 \\
\hline CDC45 & 0.02 \\
\hline KIF20A & 0.02 \\
\hline KIF14 & 0.03 \\
\hline PBK & 0.03 \\
\hline EPR1 & 0.04 \\
\hline MELK & 0.04 \\
\hline HOXD9 & 0.05 \\
\hline KIF2C & 0.05 \\
\hline BUB1B & 0.06 \\
\hline ESPL1 & 0.06 \\
\hline RRM2 & 0.06 \\
\hline HJURP & 0.07 \\
\hline TOP2A & 0.08 \\
\hline AURKB & 0.09 \\
\hline MKI67 & 0.09 \\
\hline MYBL2 & 0.09 \\
\hline \multicolumn{2}{|l|}{ Upregulated } \\
\hline C1orf115 & 0.01 \\
\hline KLK7 & 0.01 \\
\hline SULT4A1 & 0.01 \\
\hline TSHR & 0.01 \\
\hline BMP2 & 0.02 \\
\hline VEPH1 & 0.02 \\
\hline RASAL1 & 0.03 \\
\hline SNAP25 & 0.03 \\
\hline DLL3 & 0.04 \\
\hline TMEM130 & 0.04 \\
\hline HEY2 & 0.05 \\
\hline A2BP1 & 0.05 \\
\hline HIPK4 & 0.06 \\
\hline CRHR2 & 0.08 \\
\hline SPARC & 0.08 \\
\hline SLC12A5 & 0.10 \\
\hline VSNL1 & 0.10 \\
\hline
\end{tabular}

increased survival rate $(\mathrm{P}=0.10)$. $\mathrm{GO}$ annotation revealed that VSNL1 is involved in several normal neuron physiological functions. Current data also indicate that VSNAL1 may be associated with schizophrenia and frontal cortical function (29).

SULT4A1 encoded protein is a brain-specific sulfotransferase, which is widely expressed in the majority of human brain compartments and may be involved in the metabolism of neurotransmitters (30). The SULT4A1 gene, located in the frequently deleted $22 \mathrm{q} 13.3$ chromosomal region, is
Table VI. miRs associated with improved clinical outcome.

\begin{tabular}{lc}
\hline miR & P-value \\
\hline $\begin{array}{l}\text { Downregulated } \\
\text { has-miR-10b } \\
\text { has-miR-27a }\end{array}$ & 0.03 \\
Upregulated & 0.01 \\
has-miR-138-2 & \\
has-miR-138-1 & 0.04 \\
has-miR-139 & 0.02 \\
has-miR-329-1 & 0.02 \\
has-miR-412 & 0.04 \\
has-miR-431 & 0.07 \\
has-miR-495 & 0.04 \\
hsa-miR-656 & 0.10 \\
\hline miR, microRNA. & 0.09 \\
\hline
\end{tabular}

downregulated in ependymoma $(31,32)$. The present study showed that SULT4A1 was downregulated in the glioma group ( $\log 2$ foldchange $=-3.15 ; \mathrm{P}<0.05)$. In addition, a high expression of SULT4A1 was associated with increased survival rates, compared with a low expression. Therefore, SULT4A1 may be important in tumorigenesis and as a prognostic molecule in grade III gliomas.

Few studies have been performed on SNAP25 in glioma. SNAP-25 is a t-SNARE protein, which is encoded by the SNAP25 gene in humans (33). SNAP-25 is considered to account for the specificity of membrane fusion and to directly execute fusion by forming a tight complex, which brings the synaptic vesicle and plasma membranes together (34). In the present study, SNAP25 was a prognostic factor in patients with high grade glioma $(\mathrm{P}=0.03)$. The overexpression of SNAP25 predicted increased survival rates, compared with glioma patients with a lower expression of SNAP25.

A2BP1 serves to regulate the alternative splicing of TPM1 to promote cytoskeletal organization and terminal differentiation, and the loss of A2BP1 contributes to the tumorigenesis in GBM by causing compromised terminal differentiation (35). The present study found that A2BP1 was downregulated in grade III gliomas, and that a high expression level of A2BP1 was predictive of longer survival rates $(\mathrm{P}=0.05)$.

In the present study, CASC5 was identified as a prognostic factor in high grade glioma $(\mathrm{P}=0.02)$. CASC5 is a component of the kinetochore. It is involved in microtubule attachment to chromosome centromeres and in activation of the spindle checkpoint during mitosis. The CASC5 gene is upregulated in the regions of cell proliferation surrounding the ventricles during fetal brain development (36). In GBM, the expression level of CASC5 is higher, compared with that in the normal brain (37).

Current data suggests that the KLK7 protein offers potential as a prognostic marker of patient survival rates in GBM, with elevated expression levels of KLK7 associated with poor patient survival rates $(38,39)$. By contrast, the present study found that KLK7 was downregulated in glioma (foldchange=-3.98; 

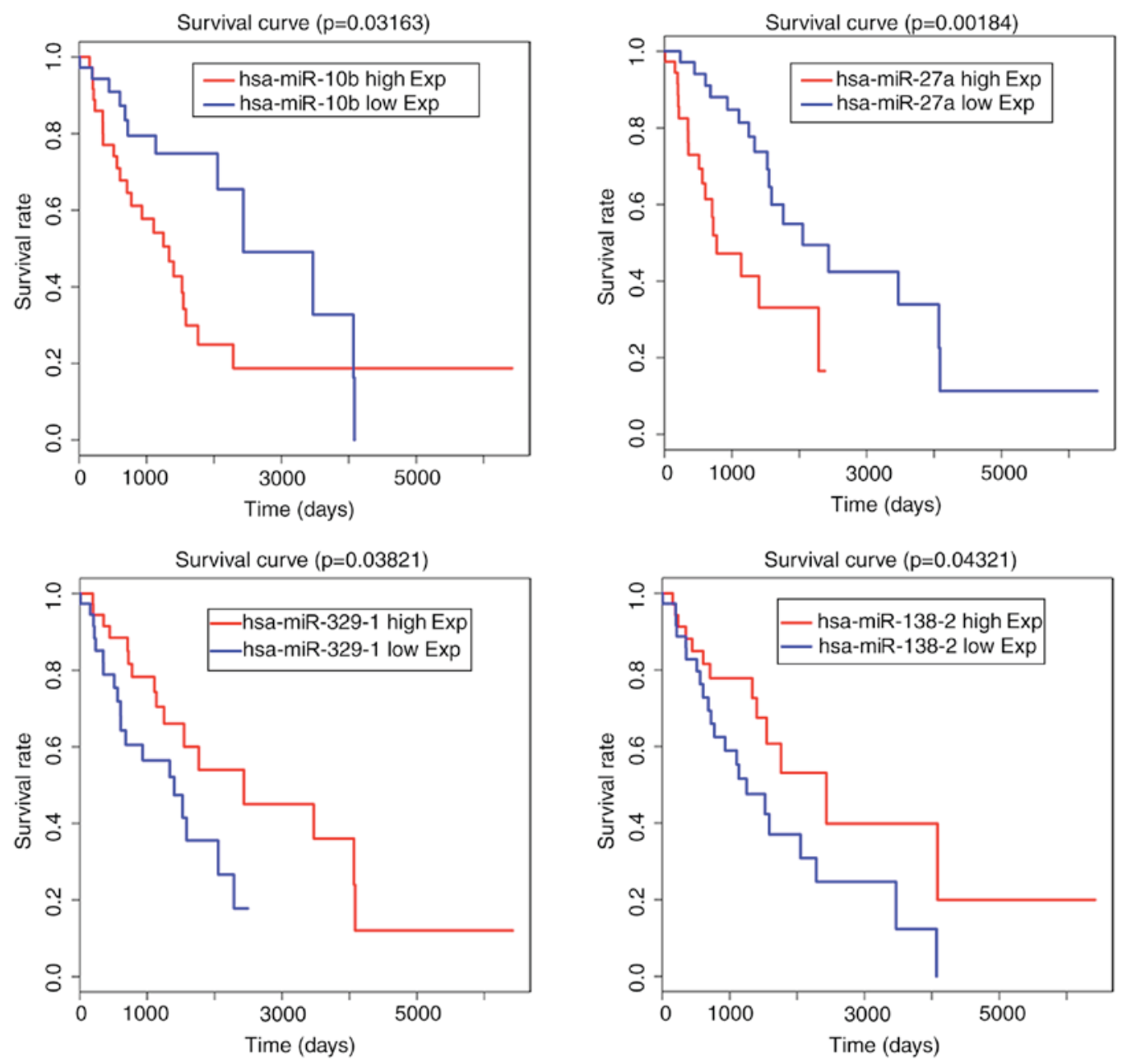

Figure 6. MicroRNAs predicting the prognosis of patients with glioma. Downregulated miR-10b and miR-27a predicted increased survival rate; upregulated miR-329-1 and miR-138-2 predicted increased survival rate. miR, microRNA; Exp, expression.

$\mathrm{P}<0.01$ ), and the decline in the expression of KLK7 was associated with poor patient survival rates in high grade glioma $(\mathrm{P}=0.01)$. Previous evidence suggests that KLK7 is differentially regulated in a variety of tumors, and is important in the normal physiology of the skin, particularly in epidermal homeostasis. The majority of evidence indicates that overexpression of KLK7 is associated with poor patient survival rates or increased tumor cell proliferation (40). However, the present study revealed that the expression of KLK7 was downregulated in prostate cancer and that the low expression was closely correlated with advanced disease stage, predictive of a poor prognosis (41). Further investigations are required in the future to identify the role of KLK7 in brain glioma.

The present studies found that BMP2, DLL3 and HEY2 were overexpressed in glioma $(42,43)$. In addition, the elevation of these neurogenesis-associated genes was associated with an increase survival rate in patients with high grade glioma. Current evidence suggests that neurogenesis-associated genes are expressed at high levels in patients with glioma, including BMP2 (43), DLL3 and HEY2, which are important in neurogenesis and may preferentially lead to the terminal differentiation of malignant cells (42).

The present study also observed that tumors with higher expression levels of HJURP were associated with poor prognosis. A previous study demonstrated that the overexpression of HJURP may be important in the maintenance of highly proliferative cells in glioma, and may be an independent prognostic factor, or a potential therapeutic target, for patients with high grade glioma (44).

According to the results of the present study, the expression of HOXD9 was markedly increased in high grade glioma, and the higher expression of HOXD9 was associated with poor survival rates in patients with glioma. HOXD9 was expressed at a low level in the normal brain, however, in glioma tissues and glioma cancer stem cells, expression was higher, compared with that in normal brain samples. Therefore, HOXD9 may be a novel marker of cell proliferation and survival rates in glioma, and a potential therapeutic target (45).

Consistent with the present study, the gene expression levels of KIF2C, KIF14, MELK and AURKB were higher in glioma samples, compared with those in normal brain tissues. The expression of these genes was associated with histopathological grades or invasiveness of glioma, and may be a candidate prognostic marker for human glioma (46-48).

It has been shown that the increased expression of miR-10b in glioma is associated with poorer prognosis (49). In the present study, has-miR-10b, has-miR-27a, has-miR-138-2, has-miR-138-1, has-miR-139, has-miR-329-1, has-miR-431, 
has-miR-495 and has-miR-656 were associated with the gliomagenesis of high grade gliomas. miR-128 and miR-129 were involved in the co-expression network, however, they were not associated with the hub genes in the network. miR-128 and miR-129, are important regulators of proliferation and can promote the apoptosis of glioma $(50,51)$. The mechanisms underlying the changes of important miRNAs in grade III glioma require further investigation.

In conclusion, using biostatistics analysis, the present study provided improved understanding of how to identify the mechanisms underlying the tumorigenesis of high grade gliomas. The results predicted that two factors involved in the glioma deterioration process, the downregulated genes and upregulated genes, are important. A number of these genes were found to be closely associated with clinical prognosis.

\section{Acknowledgements}

The authors would like to acknowledge TCGA pilot project (established by NCI and NHGRI), providing the genomic data and clinical data of LGG. This study was supported by the Lanzhou Science and Technology Bureau Project (grant no. 2013-3-27,2015-3-86), the Gansu Province Health Industry Research Project (grant no. GSWSKY-2015-58), the National Natural Science Foundation of China (grant no. 81501116) and the Doctoral Research Fund of Lanzhou University Second Hospital (grant no. ynbskyjj2015-1-02).

\section{References}

1. Barbano R, Palumbo O, Pasculli B, Galasso M, Volinia S, D'Angelo V, Icolaro N, Coco M, Dimitri L, Graziano P, et al: A miRNA signature for defining aggressive phenotype and prognosis in gliomas. PLoS One 9: e108950, 2014.

2. Pessina F, Navarria P, Cozzi L, Ascolese AM, Simonelli M, Santoro A, Tomatis S, Riva M, Fava E, Scorsetti M and Bello L: Value of surgical resection in patients with newly diagnosed grade III glioma treated in a multimodal approach: Surgery, chemotherapy and radiotherapy. Ann Surg Oncol 23: 3040-3046, 2016.

3. Haas BE, Horvath S, Pietiläinen KH, Cantor RM, Nikkola E, Weissglas-Volkov D, Rissanen A, Civelek M, Cruz-Bautista I, Riba L, et al: Adipose co-expression networks across Finns and Mexicans identify novel triglyceride-associated genes. BMC Med Genomics 5: 61, 2012.

4. Silverman EK and Loscalzo J: Network medicine approaches to the genetics of complex diseases. Discov Med 14: 143-152, 2012.

5. Zhang B and Horvath S: A general framework for weighted gene co-expression network analysis. Stat Appl Genet Mol Biol 4: Article17, 2005.

6. Chen Y, Zhu J, Lum PY, Yang X, Pinto S, MacNeil DJ, Zhang C, Lamb J, Edwards S, Sieberts SK, et al: Variations in DNA elucidate molecular networks that cause disease. Nature 452: 429-435, 2008.

7. Langfelder $\mathrm{P}$ and Horvath S: WGCNA: An R package for weighted correlation network analysis. BMC Bioinformatics 9: 559,2008

8. Voineagu I, Wang X, Johnston P, Lowe JK, Tian Y, Horvath S, Mill J, Cantor RM, Blencowe BJ and Geschwind DH: Transcriptomic analysis of autistic brain reveals convergent molecular pathology. Nature 474: 380-384, 2011.

9. Zhao W, Langfelder P, Fuller T, Dong J, Li A and Hovarth S: Weighted gene coexpression network analysis: State of the art. J Biopharm Stat 20: 281-300, 2010.

10. Cancer Genome Atlas Research Network, Weinstein JN, Collisson EA, Mills GB, Shaw KR, Ozenberger BA, Ellrott K, Shmulevich I, Sander C and Stuart JM: The cancer genome atlas pan-cancer analysis project. Nat Genet 45: 1113-1120, 2013.
11. Langfelder P and Horvath S: Eigengene networks for studying the relationships between co-expression modules. BMC Syst Biol 1: 54, 2007.

12. Ding Y, Hubert CG, Herman J, Corrin P, Toledo CM, Skutt-Kakaria K, Vazquez J, Basom R, Zhang B, Risler JK, et al: Cancer-specific requirement for BUB1B/BUBR1 in human brain tumor isolates and genetically transformed cells. Cancer Discov 3: 198-211, 2013.

13. Venere M, Miller TE and Rich JN: Mitotic control of cancer stem cells. Cancer Discov 3: 141-144, 2013.

14. Xiao YX and Yang WX: KIFC1: A promising chemotherapy target for cancer treatment? Oncotarget 7: 48656-48670, 2016.

15. Stevnsner T and Bohr VA: Studies on the role of topoisomerases in general, gene- and strand-specific DNA repair. Carcinogenesis 14: 1841-1850, 1993.

16. Watt PM and Hickson ID: Structure and function of type II DNA topoisomerases. Biochem J 303: 681-695, 1994.

17. Kasahara K, Fujiwara Y, Sugimoto Y, Nishio K, Tamura T, Matsuda T and Saijo N: Determinants of response to the DNA topoisomerase II inhibitors doxorubicin and etoposide in human lung cancer cell lines. J Natl Cancer Inst 84: 113-118, 1992.

18. Shpitz B, Bomstein Y, Zehavi T, Bernheim J, Liverant S, Kaufman Z, Buklan G and Klein E: Topoisomerase IIalpha expression in ductal carcinoma in situ of the breast: A preliminary study. Hum Pathol 31: 1249-1254, 2000.

19. Taniguchi K, Wakabayashi T, Yoshida T, Mizuno M, Yoshikawa K, Kikuchi A, Nakashima N and Yoshida J: Immunohistochemical staining of DNA topoisomerase IIalpha in human gliomas. J Neurosurg 91: 477-482, 1999.

20. Arivazhagan A, Kumar DM, Sagar V, Patric IR, Sridevi S, Thota B, Srividya MR, Prasanna K, Thennarasu K, Mondal N, et al: Higher topoisomerase 2 alpha gene transcript levels predict better prognosis in GBM patients receiving temozolomide chemotherapy: Identification of temozolomide as a TOP2A inhibitor. J Neurooncol 107: 289-297, 2012.

21. Holden JA and Townsend JJ: DNA topoisomerase II-alpha as a proliferation marker in astrocytic neoplasms of the central nervous system: Correlation with MIB1 expression and patient survival. Mod Pathol 12: 1094-1100, 1999.

22. Bie L, Zhao G, Cheng P, Rondeau G, Porwollik S, Ju Y, Xia XQ and McClelland M: The accuracy of survival time prediction for patients with glioma is improved by measuring mitotic spindle checkpoint gene expression. PLoS One 6: e25631, 2011.

23. Shichiri M, Yoshinaga K, Hisatomi H, Sugihara K and Hirata Y: Genetic and epigenetic inactivation of mitotic checkpoint genes hBUB1 and hBUBR1 and their relationship to survival. Cancer Res 62: 13-17, 2002.

24. Finetti P, Guille A, Adelaide J, Birnbaum D, Chaffanet M and Bertucci F: ESPL1 is a candidate oncogene of luminal B breast cancers. Breast Cancer Res Treat 147: 51-59, 2014.

25. Zhang C, Min L, Zhang L, Ma Y, Yang Y and Shou C: Combined analysis identifies six genes correlated with augmented malignancy from non-small cell to small cell lung cancer. Tumour Biol 37: 2193-2207, 2016.

26. Yu C, Yu J, Yao X, Wu WK, Lu Y, Tang S, Li X, Bao L, Li X, Hou Y, et al: Discovery of biclonal origin and a novel oncogene SLC12A5 in colon cancer by single-cell sequencing. Cell Res 24 : 701-712, 2014.

27. Xu L, Li X, Cai M, Chen J, Li X, Wu WK, Kang W, Tong J, To KF, Guan XY, et al: Increased expression of Solute carrier family 12 member 5 via gene amplification contributes to tumour progression and metastasis and associates with poor survival in colorectal cancer. Gut 65: 635-646, 2016.

28. Barbagallo D, Condorelli A, Ragusa M, Salito L, Sammito M, Banelli B, Caltabiano R, Barbagallo G, Zappalà A, Battaglia R, et al: Dysregulated miR-671-5p/CDR1-AS/CDR1/VSNL1 axis is involved in glioblastoma multiforme. Oncotarget 7: 4746-4759, 2016.

29. Braunewell KH, Dwary AD, Richter F, Trappe K, Zhao C, Giegling I, Schönrath K and Rujescu D: Association of VSNL1 with schizophrenia, frontal cortical function, and biological significance for its gene product as a modulator of cAMP levels and neuronal morphology. Transl Psychiatry 1: e22, 2011.

30. Liyou NE, Buller KM, Tresillian MJ, Elvin CM, Scott HL, Dodd PR, Tannenberg AE and McManus ME: Localization of a brain sulfotransferase, SULT4A1, in the human and rat brain: An immunohistochemical study. J Histochem Cytochem 51: 1655-1664, 2003. 
31. Modena P, Lualdi E, Facchinetti F, Veltman J, Reid JF, Minardi S, Janssen I, Giangaspero F, Forni M, Finocchiaro G, et al: Identification of tumor-specific molecular signatures in intracranial ependymoma and association with clinical characteristics. J Clin Oncol 24: 5223-5233, 2006.

32. Dubuc AM, Northcott PA, Mack S, Witt H, Pfister S and Taylor MD: The genetics of pediatric brain tumors. Curr Neurol Neurosci Rep 10: 215-223, 2010.

33. Maglott DR, Feldblyum TV, Durkin AS and Nierman WC: Radiation hybrid mapping of SNAP, PCSK2, and THBD (human chromosome 20p). Mamm Genome 7: 400-401, 1996.

34. Rizo J and Südhof TC: Snares and Munc18 in synaptic vesicle fusion. Nat Rev Neurosci 3: 641-653, 2002.

35. Hu J, Ho AL, Yuan L, Hu B, Hua S, Hwang SS, Zhang J, Hu T, Zheng H, Gan B, et al: From the cover: Neutralization of terminal differentiation in gliomagenesis. Proc Natl Acad Sci USA 110: 14520-14527, 2013.

36. Genin A, Desir J, Lambert N, Biervliet M, Van Der Aa N, Pierquin G, Killian A, Tosi M, Urbina M, Lefort A, et al Kinetochore KMN network gene CASC5 mutated in primary microcephaly. Hum Mol Genet 21: 5306-5317, 2012.

37. Akiyama $Y$, Komiyama M, Miyata $\mathrm{H}$, Yagoto M, Ashizawa $\mathrm{T}$, Iizuka A, Oshita C, Kume A, Nogami M, Ito I, et al: Novel cancer-testis antigen expression on glioma cell lines derived from high-grade glioma patients. Oncol Rep 31: 1683-1690, 2014

38. Prezas P, Scorilas A, Yfanti C, Viktorov P, Agnanti N, Diamandis E and Talieri M: The role of human tissue kallikreins 7 and 8 in intracranial malignancies. Biol Chem 387: 1607-1612, 2006.

39. Drucker KL, Gianinni C, Decker PA, Diamandis EP and Scarisbrick IA: Prognostic significance of multiple kallikreins in high-grade astrocytoma. BMC Cancer 15: 565, 2015.

40. Walker F, Nicole P, Jallane A, Soosaipillai A, Mosbach V, Oikonomopoulou K, Diamandis EP, Magdolen V and Darmoul D: Kallikrein-related peptidase 7 (KLK7) is a proliferative factor that is aberrantly expressed in human colon cancer. Biol Chem 395: 1075-1086, 2014.

41. Zhang CY, Zhu Y, Rui WB, Dai J and Shen ZJ: Expression of kallikrein-related peptidase 7 is decreased in prostate cancer. Asian J Androl 17: 106-110, 2015.

42. Freije WA, Castro-Vargas FE, Fang Z, Horvath S, Cloughesy T, Liau LM, Mischel PS and Nelson SF: Gene expression profiling of gliomas strongly predicts survival. Cancer Res 64: 6503-6510, 2004
43. Zhang X, Wu J, Li X, Fu L, Gao D, Bai H and Liu X: Effects of recombinant human bone morphogenic protein-2 and hyaluronic acid on invasion of brain glioma in vivo. Zhonghua Yi Xue Za Zhi 82: 90-93, 2002 (In Chinese).

44. Valente V, Serafim RB, de Oliveira LC, Adorni FS, Torrieri R, Tirapelli DP, Espreafico EM, Oba-Shinjo SM, Marie SK, Paçó-Larson ML and Carlotti CG Jr: Modulation of HJURP (Holliday Junction-Recognizing Protein) levels is correlated with glioblastoma cells survival. PLoS One 8: e62200, 2013.

45. Tabuse M, Ohta S, Ohashi Y, Fukaya R, Misawa A, Yoshida K, Kawase T, Saya H, Thirant C, Chneiweiss H, et al: Functional analysis of HOXD9 in human gliomas and glioma cancer stem cells. Mol Cancer 10: 60, 2011.

46. Bie L, Zhao G, Wang YP and Zhang B: Kinesin family member 2C (KIF2C/MCAK) is a novel marker for prognosis in human gliomas. Clin Neurol Neurosurg 114: 356-360, 2012.

47. Gu C, Banasavadi-Siddegowda YK, Joshi K, Nakamura Y, Kurt H, Gupta S and Nakano I: Tumor-specific activation of the C-JUN/MELK pathway regulates glioma stem cell growth in a p53-dependent manner. Stem Cells 31: 870-881, 2013.

48. Wang Q, Wang L, Li D, Deng J, Zhao Z, He S, Zhang Y and Tu Y: Kinesin family member 14 is a candidate prognostic marker for outcome of glioma patients. Cancer Epidemiol 37: 79-84, 2013.

49. Zhang X, Cheng J, Fu L and Li Q: Overexpression of tissue microRNA10b may help predict glioma prognosis. J Clin Neurosci 29: 59-63, 2016.

50. Chen X, Zhang Y, Shi Y, Lian H, Tu H, Han S, Yin J, Peng B, Zhou B, He X and Liu W: MiR-129 triggers autophagic flux by regulating a novel Notch-1/E2F7/Beclin-1 axis to impair the viability of human malignant glioma cells. Oncotarget 7 : 9222-9235, 2016.

51. Shang C, Hong Y, Guo Y, Liu YH and Xue YX: miR-128 regulates the apoptosis and proliferation of glioma cells by targeting RhoE. Oncol Lett 11: 904-908, 2016. 